\title{
MULTIDIMENSIONAL ROUGHNESS CHARACTERIZATION FOR MICROWAVE REMOTE SENSING APPLICATIONS USING A SIMPLE PHOTOGRAMMETRIC ACQUISITION SYSTEM
}

\author{
Philip Marzahn $^{1}$, Dirk Rieke-Zapp ${ }^{2}$, Urs Wegmuller ${ }^{3}$ and Ralf Ludwig ${ }^{1}$ \\ ${ }^{1}$ Department of Geography \\ Ludwig-Maximilians University of Munich \\ Luisenstrasse 37, 80333 Munich, Germany \\ p.marzahn@iggf.geo.uni-muenchen.de \\ ${ }^{2}$ Institute of Geological Science \\ University of Bern \\ Baltzerstrasse 1+3, CH-3012 Bern, Schwitzerland \\ ${ }^{3}$ Gamma Remote Sensing \\ Worbstr. 225, CH-3073 Guemlingen, Schwitzerland
}

Commission V/6

KEY WORDS: Soil Surface Roughness, Photogrammetry, SAR, Directional Scattering, Detrending, Statistics

\begin{abstract}
:
Soil surface roughness, as investigated in this study, is a critical parameter in microwave remote sensing. As soil surface roughness is treated as a stationary single scale isotropic process in most backscattering models, the overall objective of this study was to better understand the role of soil surface roughness in the context of backscattering. Therefore a simple photogrametric acquisition setup was developed for the characterization of soil surface roughness. In addition several suited SAR images of different sensors (ERS-2 and TerraSAR-X) were acquired to quantify the impact of soil surface roughness on the backscattered signal. Major progress achieved in this work includes the much improved characterization of in-field soil surface roughness. Good progress was also made in the understanding of backscattering from bare surface in the case of directional scattering.
\end{abstract}

\section{INTRODUCTION}

Micro scale soil surface roughness is a critical parameter in a wide range of environmental applications. In microwave remote sensing, it is well known that micro scale soil surface roughness has a considerable impact on the backscatter signal. Without considering roughness terms in several retrieval algorithms, the derivation of geo-physical variables will lead to insufficient results or in a general over- or underestimation of the desired variables. Despite this, recent studies have shown, the periodical component of soil surface roughness and its orientation cause significant backscatter differences in different SAR images acquired with short temporal intervals under slightly different aspect angles (Wegmuller et al., 2011). However, for an appropriate characterization of in-situ soil surface roughness measurements, the default measurement devices are still not sufficient in accuracy (mesh boards) or in the acquisition speed (laser profiler). Thus, precise roughness measurements with a statistically robust acquisition size are so far rarely available, leading to a generalized roughness representation in recent backscatter models. In such models, soil surface roughness is generally treated as single scale stationary isotropic process. Indeed, as soil surface roughness in an agricultural environment can be considered as multi-scale and anisotropic, a three-dimensional acquisition scheme is required to accomplish theses requirements (Marzahn et al., 2012a,b). In this paper we present a simple acquisition setup to acquire soil surface roughness and show the impact of soil surface roughness on microwave backscattering from agricultural fields in the special case of directional scattering. Datasets were acquired at the Wallerfing test site, which is part of the SMOS Cal/Val Upper Danube test site (Schlenz et al., 2010).

\section{METHODS}

Several field campaigns were scheduled in conjunction with ERS2 and TerraSAR-X acquisitions over the Wallerfing test site which is part of the SMO Cal/Val activities (Schlenz et al., 2010) located in the Upper Danube watershed approx. $100 \mathrm{~km}$ northeast of Munich. The region, which has a low relief energy, is mainly agricultural in character and the main crops are winter wheat, winter barley, corn and sugar beet. During the campaign most of the crops had been sown already and were already at the beginning of their growth. The seedbed structure was at all sample points still well developed due to the lack of precipitation. Thus, the sample points (elementary sample units, ESU) all represent an already prepared seedbed pattern. Table 1 summarizes the characteristics of the sample points. In addition to the roughness measurements, soil moisture, vegetation height and coverage, row orientation and linearity as well as row distance were measured. For the entire test site a landuse map was available.

\subsection{Roughness Acqusition Setup}

For measuring soil surface roughness a simple photogrametric approach was chosen. Therefore the setup consists of a customized Canon EOS 5D used with a Canon EF 2/35 mm lens and a reference frame. As the performance of such consumer grade camera is usually limited by their sensors and lens stability (Rieke-Zapp and Nearing, 2005), the lens was fixed by placing epoxy resin between the focusing tube and the outer lens tube, reducing the maximum absolute LME to $47 \mu \mathrm{m}$ (Rieke-Zapp et al., 2009). After fixation of the lens, the camera was calibrated and its interior orientation parameters estimated by Aicon 3D Studio and converted for further usage in Leica Photogrametry Suite (LPS). Parameters of the interior orientation are provided in Marzahn et 


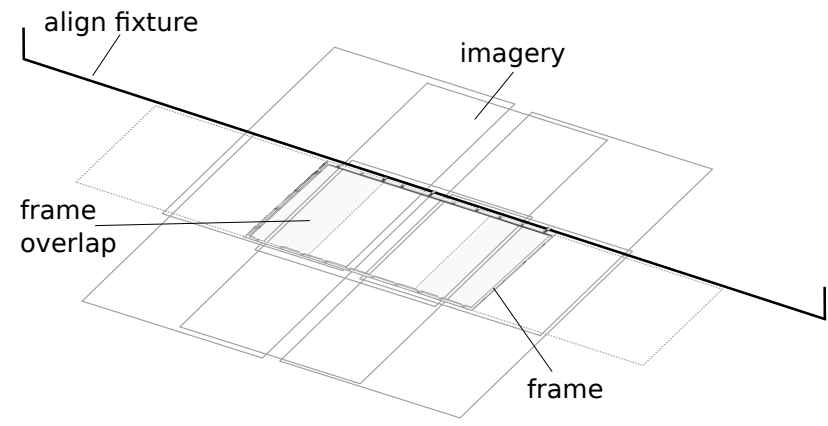

Figure 1: Roughness acquisition scheme and image arrangement for stereo coverage of the frame

\begin{tabular}{|c||c||c||c|}
\hline ESU & Landuse & surface type & size $\left[\mathrm{m}^{2}\right]$ \\
\hline \hline R11 & maize & seedbed & 6 \\
\hline R12 & onion & smooth crusted & 6 \\
\hline R13 & sugarbeet & crusted seedbed & 8 \\
\hline R14 & sugarbeet & crusted seedbed & 8 \\
\hline R21 & sugarbeet & crusted seedbed & 6 \\
\hline R22 & sugarbeet & crusted seedbed & 6 \\
\hline R23 & sugarbeet & crusted seedbed & 6 \\
\hline R30 & winter rape & seedbed & 22 \\
\hline
\end{tabular}

Table 1: Characteristics of sample points acquired within this study

al. (2012a). As LPS needs for establishing the exterior orientation Ground Control Points (GCPs), a reference frame in the size of $1 \times 2.5 \mathrm{~m}^{2}$ was built providing 28 GCPs with a vertical accuracy of $0.1 \mathrm{~mm}$ measured with a caliper ruler. As the frame is limited in its size, it is necessary, for larger roughness acquisitions, to acquire consecutive image acquisitions of the frame by moving it along a levelled plane which is ensured by an align fixture (see Fig. 1). During post processing, the individual DSMs were merged to a final large DSM by image matching techniques.

In this study, roughness measurements were made over several bare or sparse vegetated fields. Over the latter ones, the vegetation was carefully removed from the scene without disturbing the soil's surface. For each sample point acquisitions were made parallel and perpendicular to the row direction of the agricultural fields. Table 1 summarizes the characteristics of the sample points where soil surface roughness was measured.

\subsection{Roughness Characterization}

Soil surface roughness can be characterised by a vertical and a horizontal component. Thus, to quantify soil surface roughness, two different roughness indices where chosen to numerically describe soil surface roughness. To quantify the vertical component, the RMS height $s$ was chosen, which is defined as:

$$
s[c m]=\sqrt{\frac{\sum_{i=1}^{n}\left(Z_{i}-\bar{Z}\right)^{2}}{n-1}}
$$

and describes the standard deviation of the heights $(Z)$ to a reference height $(\bar{Z})$. The horizontal roughness component is described by the autocorrelation length $l$. For an efficient estimation of $l$, a variogram analysis was used and inverted the autocorrelation function (ACF) from a calculated theoretical directional var$\operatorname{iogram}\left(\tilde{\gamma}\left(h_{\vec{j}}\right)\right)$, where $l$ is defined as the distance $(h)$ at which the ACF drops under $e^{-1}$ (Blaes and Defourny, 2008). This implies an exponential fit of the theoretical variogram and therefore of the ACF.

The theoretical variogram $(\tilde{\gamma})$ with an exponential shape is fitted to a directional experimental variogram $\left(\hat{\gamma}\left(h_{\vec{j}}\right)\right)$, which is defined as (Webster and Oliver, 2007):

$$
\hat{\gamma}\left(h_{\vec{j}}\right)=\frac{1}{2 n} \sum_{i=1}^{n}\left[Z\left(x_{i}\right)-Z\left(x_{i}+h_{\vec{j}}\right)\right]^{2}
$$

From the theoretical variogram $(\tilde{\gamma})$ the $\operatorname{ACF}(\tilde{\rho})$ can be derived as follows:

$$
\tilde{\rho}(h)=1-\frac{\tilde{\gamma}(h)}{\tilde{\gamma}(\infty)}
$$

where $\tilde{\gamma}(h)$ is the semi variance at distance $h$ between two points and $\tilde{\gamma}(\infty)$ is the semi variance at distance where the sill of the variogram is reached. For the assumed exponential model, where the sill is asymptotically approached, $\tilde{\gamma}(\infty)$ corresponds to the distance where $95 \%$ of the sill is reached (Blaes and Defourny, 2008).

To characterize the anisotropic pattern of a corresponding soil surface, the directional autocorrelation length was calculated for direction between $45^{\circ}$ and $135^{\circ}$ with a $1^{\circ}$ interval.

\subsection{Decomposing Soil Surface Roughness}

From Figure 2a and its corresponding sample variogram (Fig. 3a) it is obvious that a two scale roughness pattern can be observed on an agriculturally formed soil surface. As different roughness scales have an impact on the backscattered signal in microwave remote sensing, it is important to characterize both scales. Therefore, again variography is used to decompose and characterize the soil surface at different scales. As the variogram shows a surface inherent behaviour with strong similarities at distances in range of $180 \mathrm{~cm}$, which correspond to the wheel tracks in Figure 2a, a two scale roughness pattern is indicated. Thus, the variogram for the whole sample plot describes the semi-variance of the roughness pattern which is strongly imposed by the large scale roughness pattern (e.g. wheel tracks of drilling machine). To characterize the small scale roughness pattern (e.g. seedbed rows, soil clod distribution) we defined a threshold to mask out the wheel tracks and calculated variograms for each surface again. As a result, for each roughness scale a roughness index is calculated.

\subsection{Detrending}

As can be seen in Figure $2 b$ and $3 b$ several DSMs showed a spatial trend in elevation due to higher order topographic patterns such as general slope effects. Lievens et al. (2009) described the importance of detrending the data and the effects of different detrending procedures on the retrieved roughness indices. In this study, two detrending models have been defined which can be described by:

$$
Z_{\text {mod }} \sim m X+b
$$

for the linear model and

$$
Z_{\text {mod }} \sim b+m_{1} X+m_{2} X^{2}+m_{3} X^{3}+\ldots m_{n} X^{n}
$$

for the polynominal model, where $m$ and $b$ represent the regression coefficients slope and intercept and $X$ the $\mathrm{x}$-coordinate of the sample DSM. The detrended surface $Z_{\text {res }}$ then is defined as:

$$
Z_{\text {res }}=Z-Z_{\text {mod }}
$$

\subsection{SAR Data}

Six ERS-2 and TerraSAR-X (TSX) datasets with varying Doppler centroids were acquired during the campaign in spring 2011 at 


\begin{tabular}{|c||c||c||c||c||}
\hline Date & Sensor & Dop. Centroid $[\mathrm{Hz}]$ & Mode & Pol \\
\hline \hline 20110402 & ERS & -2427.0 & Image & VV \\
\hline 20110423 & ERS & 160.0 & Image & VV \\
\hline 20110426 & ERS & 752.0 & Image & VV \\
\hline 20110507 & TSX & -9.8 & Stripmap & VV/HV \\
\hline 20110514 & ERS & -3598.0 & Image & VV \\
\hline 20110523 & ERS & 7.0 & Image & VV \\
\hline
\end{tabular}

Table 2: Acquired SAR imagery and corresponding characteristics

\begin{tabular}{|l||c||c||c||c||c||c||c|}
\hline R11 & R12 & R13 & R14 & R21 & R22 & R23 & R30 \\
\hline \hline 0.17 & 0.13 & 0.05 & 0.07 & 0.05 & 0.06 & 0.02 & 0.22 \\
\hline
\end{tabular}

Table 3: Root mean square error in $\mathrm{cm}$ of generated DSM height values compared to the manually measured GCPs for each sample point

the beginning of the growing season for the major crops in the test site. Table 2 summarizes the main characteristics of the acquisitions, while Figure 4 show as an example the backscattering in an ERS-2 image over the test site.

To detect a potential directional scattering over several agricultural fields, a SAR image is split into five sub looks according to Wegmuller et al. (2011) with a 50 percent overlap and HSI images are generated, where hue corresponds to the backscatter ratio of two sub looks, saturation to the backscatter change and intensity to the backscattering in the first image of the pair. Figure 5 shows as an example a HSI composite for the 23 of May 2011.

\section{RESULTS AND DISCUSSION}

\subsection{Roughness characterization}

Table 3 shows the root mean square error in the Z-direction ( $\mathrm{RMSE}_{\mathrm{Z}}$ ) of the sample plots. The results show a high accuracy of the generated DSMs compared to the manually measured checkpoints installed on the reference frame, thus providing a robust basis for the characterization of soil surface roughness statistics.

Figures 2 and 3 show as an example three generated DSM with typical problems occurring when measuring soil surface roughness in an agricultural environment. All DSMs show a multi scale roughness pattern ranging from small (micro) scale roughness pattern comprising the soil clods and seedbed rows over meso scale roughness (wheel tracks) and large scale roughness pattern such as general slope effects. Table 4 summarizes the characterization of the roughness spectra by using the defined roughness indices for the micro $\left(s_{1}\right.$ and $\left.l_{1}\right)$ and the meso roughness scale $\left(s_{2}\right.$ and $l_{2}$ ) separately and the output from the detrending procedure. By analysing the effects of detrending it is obvious that for the RMS height no significant change is observed due to the detrending procedure. Results for the autocorrelation length $l$ change significantly in the order of several decimetres. Except for R30, a $22 \mathrm{~m}^{2}$ large sample plot, a significant change in $s$ can be observed, due to the strong topographic influence with a range in heights of $20 \mathrm{~cm}$. To model this strong trend a polynomial approach of 9th order was chosen.

Figure 3a indicates a significant two-scale roughness pattern for sample plot R12. Different points with a certain distance in range of $200 \mathrm{~cm}$ to each other show a strong similarity, thus indicating periodicity in the soil surface roughness pattern with a range of $200 \mathrm{~cm}$. From Figure 2a it is obvious that this pattern is clearly related to the wheel tracks of the tillage machines used during
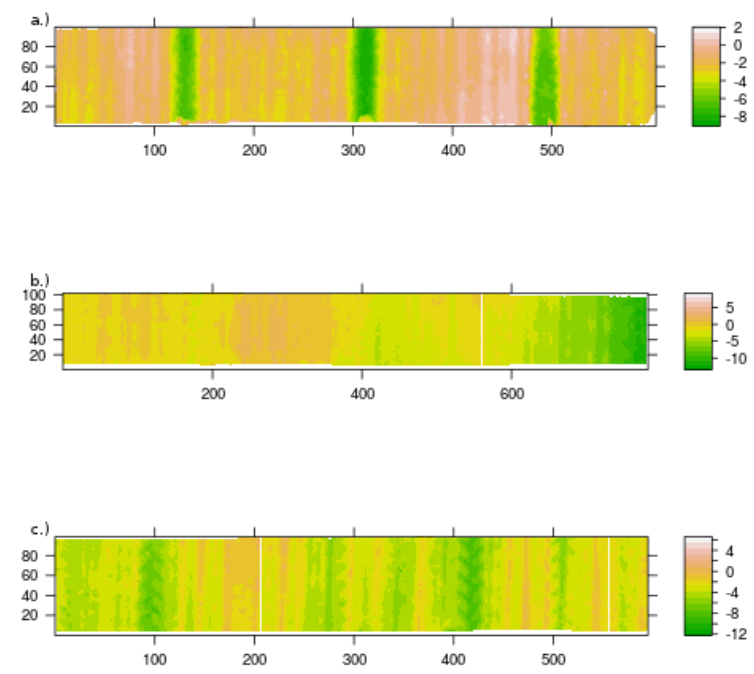

Figure 2: Three sample DSMs of different roughness plots showing a.) a significant two scale roughness pattern (R12), b.) a spatial trend (R14) and c.) no spatial trend with an insignificant two scale roughness pattern (R21). Units in $\mathrm{cm}$

\begin{tabular}{|c||c||c||c|c|}
\hline ESU & $s_{1}$ & $s_{2}$ & $l_{1}$ & $l_{2}$ \\
\hline \hline R11 & 0.88 & 1.84 & 11.0 & 29.5 \\
\hline R12 & 0.85 & 1.73 & 71.6 & 38.27 \\
\hline R13 & $1.15(1.09)$ & $2.43(2.84)$ & $38.3(17.7)$ & $53.04(69.05)$ \\
\hline R14 & $0.24(0.86)$ & $2.26(2.5)$ & $41.01(96.39)$ & $98.04(169.14)$ \\
\hline R21 & 1,24 & 1.45 & 31.1 & 38.52 \\
\hline R22 & $0.77(0.93)$ & $1.12(1.51)$ & $20.5(26.4)$ & $23.47(55.4)$ \\
\hline R23 & $1.08(1.31)$ & $2.38(2.71)$ & $27.3(25.7)$ & $105.6(107.5)$ \\
\hline R30 & $1.18(2.84)$ & $1.19(3.26)$ & $17.2(145.7)$ & $17.2(359.52)$ \\
\hline
\end{tabular}

Table 4: Results of two scale roughness representation. In brackets the results for the non detrended original surfaces. Units in $\mathrm{cm}, s_{1}, l_{1}$ correspond to the small scale roughness pattern, $s_{2}, l_{2}$ to the large scale roughness pattern 

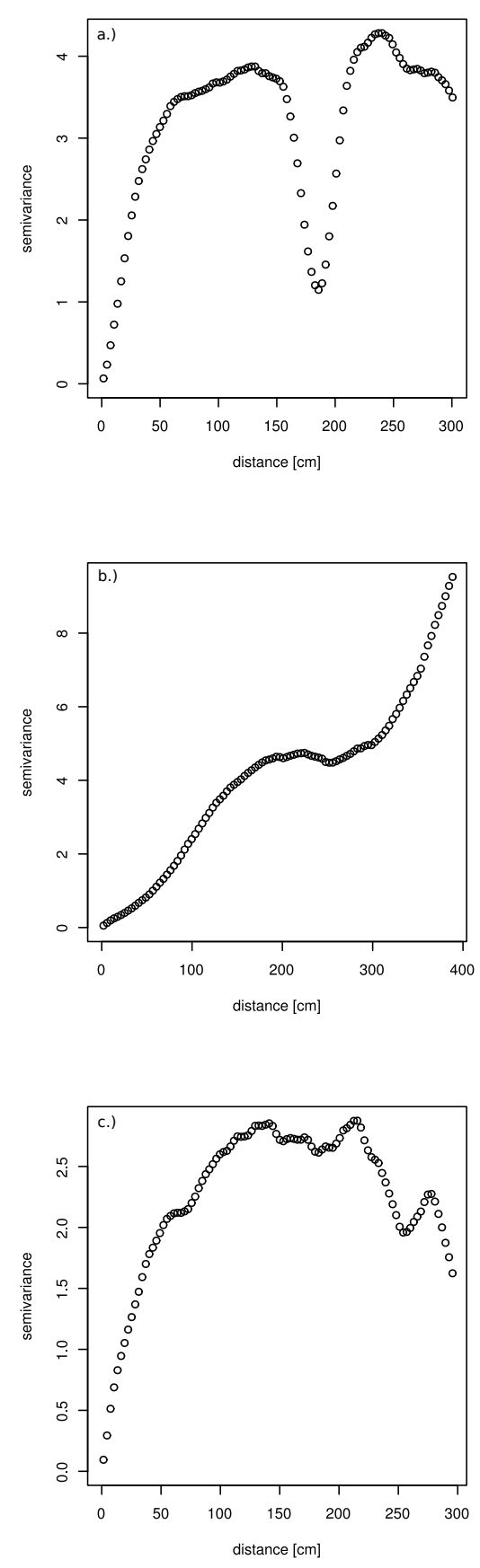

Figure 3: Sample variograms of the roughness plots from Fig 2 showing a.) a significant two scale roughness pattern (R12), b.) a spatial trend (R14) and c.) no spatial trend with an insignificant two scale roughness pattern (R21)

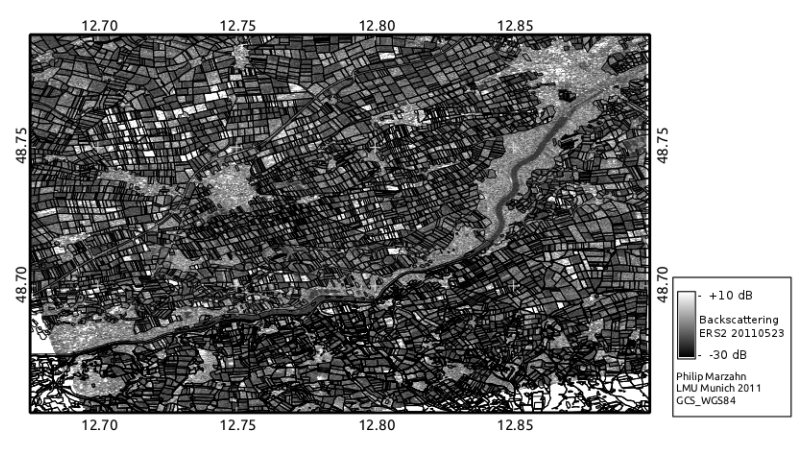

Figure 4: ERS-2 scene, acquired at the 23 of May 2011, over the Wallerfing test site, indicating strong directional backscattering over several fields in the north-western part of the image

seedbed preparation. Thus the sharply bounded wheel tracks with a height difference of 4-6 $\mathrm{cm}$ to the surrounding seedbed biases the characterization of the roughness indices. All sample plots show this two scale roughness representation, due to the availability of a seedbed structure imposed over wheel tracks, at which for the small scale roughness pattern the values for $s_{l}$ and $l_{l}$ are lower than for the large scale roughness pattern. For sample plot R12, which represents a smooth crusted onion field, the autocorrelation length for the small scale roughness pattern is higher than the large scale roughness pattern, indicating a very smooth surface with sharply bounded wheel tracks (Fig. 2a). It should be highlighted, that even under the same land use type (e.g. sugar beet) the roughness values indicate different roughness conditions, which are a result of the different tillage machines used and the state of crusting. Sample plots R14 and R21 illustrate this effect, both represent sugar beet fields at the same crusted stage. In contrast, sample plot R30 shows no significant two scale roughness process, which is due to the missing presence of wheel tracks or other higher order roughness patterns and thus roughness is only defined by the present seedbed structure. As a result, the values of $s$ and $l$ are equal for both scales.

\subsection{SAR data and the identifying of directional scattering}

Figure 4 reveals a strong backscatter for several fields in the northwestern part of the scenes, indicating either vegetated fields as well as man made structures or directional scattering from bare fields (Wegmuller et al., 2011). The HSI image with an Doppler difference of $1000 \mathrm{~Hz}$, which correspond to a difference in the look vector of $0.5^{\circ}$, indicate that those fields are characterized by directional scattering. Those fields are mostly bare recently seedbed prepared or sparsely vegetated fields, with the row pattern orientated quasi perpendicular to the sensors look vector with a difference of $\pm 5^{\circ}$. Wegmuller et al. (2011) found similar observations over the Flevoland test site. Comparing those fields with the neighbouring fields, with a different row orientation but same landuse type and phenology, the backscattering is up to $10 \mathrm{~dB}$ higher. Thus, a directional backscattering, which mainly originates from the row orientation can be observed, however there are several differences between different fields (see Fig. 4) which only differ in the observed roughness conditions.

\subsection{Impact of soil surface roughness on backscattering}

Figure 6 shows the directional backscattering in $\mathrm{dB}$ over bare or sparsely vegetated fields in dependency of the row orientation to the sensors look vector and the roughness conditions at the time of each image acquisition. As one can clearly observe, there is 


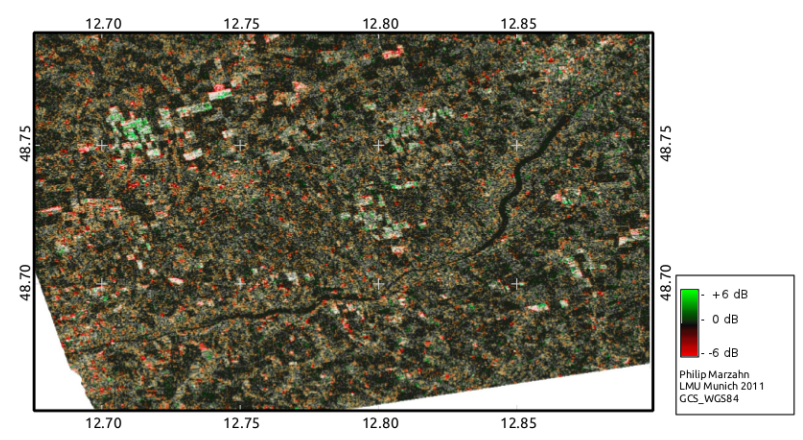

Figure 5: HSI (HueSaturationIntensity) composite of the backscatter ratio (hue), backscatter change (saturation) and backscattering in the first image of the pair (intensity) over Wallerfing site on 23 of May 2011. Backscatter ratio (hue) ranges from 6 to $+6 \mathrm{~dB}$; mean intensity (intensity) from $22 \mathrm{~dB}$ to + $6 \mathrm{~dB}$; absolute backscatter change (saturation) from 0 to $+6 \mathrm{~dB}$. Red indicates higher intensity for the sub look 1 , and green higher intensity for sub look 5 .

a strong first order dependency of the directional scattering from the row orientation which was also observed by Wegmuller et al. (2011). However, a secondary dependency can be observed from the roughness conditions. Thus, the first order dependency can be described by a Gaussian model (Mattia, 2011; Wegmuller et al., 2011) with a peak at rows orientated perpendicular to the sensors look vector (in Figure 6, perpendicular $=0^{\circ}$ ). However, for sample points R12 and R21 the backscattering is significantly higher compared to the other sample points. This could be related to the roughness conditions measured at both sample points, as both show the same roughness values for the meso scale roughness pattern with an autocorrelation length of approx. $38 \mathrm{~cm}$. In contrast, for the TSX data, no significant dependency of the directional scattering from the measured roughness conditions could be observed, thus it is only dependent from the row orientation. Following the study of Lievens et al. (2009), detrending of roughness measurements has a strong impact of the calculated roughness indices. As mentioned above, this effect is mainly present for the autocorrelation length. Indeed, the different scales of soil surface roughness, which were reduced by detrending to a single scale, have an impact on microwave backscattering. From Figure 6 one could observe that sample point R13 has a significantly lower backscatter in every SAR acquisition than samples R12 and R21, even when detrending reveals the same autocorrelation length. Looking closely to the non-detrended roughness values of R13, the surface shows a general slope and as a consequence lowering and disturbing the superimposition of the micro and meso scale roughness component to a comparable strong directional backscatter .

\section{CONCLUSIONS AND FUTURE WORK}

In this paper we proposed a simple and efficient approach for measuring multi dimensional soil surface roughness in an agricultural environment for microwave remote sensing applications. A customized Canon EOS 5D was used to generate and provide highly accurate digital surface models. With a vertical displacement of $\mathrm{RMSE}_{\mathrm{z}} \leq 0.2 \mathrm{~mm}$, the technique is suited for the characterization of soil surface roughness for microwave remote sensing studies (Lievens et al., 2009). In this study we proposed an approach to decompose soil surface roughness in its different scales, ranging from micro scale (soil clods and seedbed rows)

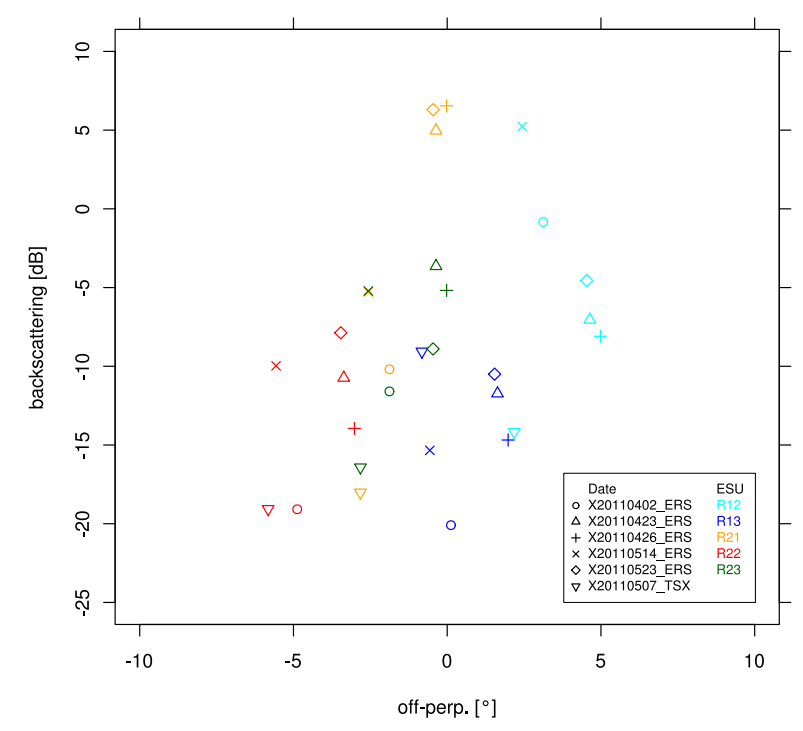

Figure 6: Backscattering $[\mathrm{dB}]$ versus row direction $\left(0^{\circ}=\right.$ rows perpendicular to the sensors look vector) for the roughness sample plots

over meso scale soil surface roughness (wheel tracks) to a macro scale component (general slope, formed by the landscape). As each scale has its contributions to microwave backscattering, the effect of detrending on soil surface roughness measurements has to reconsidered. From the roughness measurements made in this study one can obtain that for the detrended data, several samples yield the same roughness values (e.g. R13) as for other samples without detrending (e.g. R12/R21). However, under the same conditions (soil moisture, row orientation, phenology) the backscattering is significantly lower than for the non detrended samples, leading to the assumption that using detrended roughness measurements biases a potential backscatter model. Indeed, this conclusion has to be approved by further investigations. In the context of "Flashing Fields", which are characterised by a strong directional scatter caused by the seedbed rows orientation to the sensors look vector, we confirmed the investigations of Wegmuller et al. (2011) and Mattia (2011). However, we also identified a second order dependency of the flashing by the soil surface roughness conditions. From this study, it is to conclude that for a C-Band sensor such as ERS-2 an autocorrelation length of approx. $38 \mathrm{~cm}$ significantly increases the flashing effect in microwave remote sensing.

Future work will comprise an in depth analysis of the effect of detrending on roughness values and their impact on SAR backscatter. In addition further "Flashing Fields" will be analysed with a focus on the impact from soil surface roughness and the impact of the different roughness scales.

\section{ACKNOWLEDGEMENTS}

The authors would like to thank the numerous student assistants help during the field campaigns and during the post processing of the DSMs. In addition the authors would like to thank "Photogrammetrie Perrinjaquet AG" Guemmlingen, Switzerland, for the fixation of the Canon lens. 


\section{References}

Blaes, X. and Defourny, P., 2008. Characterizing bidimensional roughness of agricultural soil surfaces for SAR modeling. Geoscience and Remote Sensing, IEEE Transactions on 46(12), pp. 4050-4061.

Lievens, H., Vernieuwe, H., Alvarez-Mozos, J., De Baets, B. and Verhoest, N., 2009. Error in radar-derived soil moisture due to roughness parameterization: An analysis based on synthetical surface profiles. Sensors 9(2), pp. 1067-1093.

Marzahn, P., Rieke-Zapp, D. H. and Ludwig, R., 2012a. Assessment of soil surface roughness statistics for microwave remote sensing applications using a simple photogrammetric acquisition system. ISPRS Journal of Photogrammetry and Remote Sensing submitted, pp. submitted.

Marzahn, P., Seidel, M. and Ludwig, R., 2012b. Decomposing dual scale soil surface roughness for microwave remote sensing applications. Remote Sensing submitted, pp. submitted.

Mattia, F., 2011. Coherent and incoherent scattering from anisotropic tilled soil surfaces. Waves in Random and Complex Media 21(2), pp. 278300.

Rieke-Zapp, D. and Nearing, M., 2005. Digital close range photogrammetry for measurement of soil erosion. Photogrammetric Record 20(109), pp. 69-87.

Rieke-Zapp, D., Tecklenburg, W., Peipe, J., Hastedt, H. and Haig, C., 2009. Evaluation of the geometric stability and the accuracy potential of digital cameras - comparing mechanical stabilisation versus parameterisation. ISPRS Journal of Photogrammetry and Remote Sensing 64(3), pp. 248-258.

Schlenz, F., Dall'Amico, J., T., Loew, A. and Mauser, W., 2010. Smos validation in the upper danube catchment (udc): A status report eigth month after launch. In: Proceedings of ESA LIving Planet Symposium 2010 Bergen, Norway.

Webster, R. and Oliver, M. A., 2007. Geostatistics for Environmental Scientists. 2 edn, John Wiley /\& Sons, LTD

Wegmuller, U., Santoro, M., Mattia, F., Balenzano, A., Satalino, G., Marzahn, P., Ludwig, R. and Floury, N., 2011. Progress in the understanding of narrow directional microwave scattering of agricultural fields. Remote Sensing of Environment 115, pp. 2423-2433. 